\title{
NUMERICAL SOLUTIONS OF THE COMPLEX LANGEVIN EQUATIONS IN POLYMER FIELD THEORY*
}

\author{
ERIN M. LENNON ${ }^{\dagger}$, GEORGE O. MOHLER $\ddagger$, HECTOR D. CENICEROS§ ${ }^{\S}$ CARLOS J. \\ GARCÍA-CERVERA ฯ, AND GLENN H. FREDRICKSON"
}

\begin{abstract}
Using a diblock copolymer melt as a model system, we show that complex Langevin (CL) simulations constitute a practical method for sampling the complex weights in field theory models of polymeric fluids. Prior work has primarily focused on numerical methods for obtaining mean-field solutions - the deterministic limit of the theory. This study is the first to go beyond EulerMaruyama integration of the full stochastic CL equations. Specifically, we use analytic expressions for the linearized forces to develop improved time integration schemes for solving the nonlinear, nonlocal stochastic CL equations. These methods can decrease the computation time required by orders of magnitude. Further, we show that the spatial and temporal multiscale nature of the system can be addressed by the use of Fourier acceleration.
\end{abstract}

Key words. diblock copolymer, Langevin equation, stochastic simulation

AMS subject classifications. $65 \mathrm{Z} 05,60 \mathrm{H} 35$

1. Introduction. Systems with mesoscopic ordering on scales of $1 \mathrm{~nm}$ to $1 \mu \mathrm{m}$ have proven vital to the development of novel polymeric materials. As modeling on these length scales is not tractable with either molecular or macroscopic simulations, there has been much interest in using techniques of statistical field theory to build coarse-grained models of polymers that self-assemble on the mesoscale. In this approach, a particle-based model is transformed into a classical statistical field theory in which particle-particle interactions are replaced with particle-field interactions. Thus the degrees of freedom associated with individual particles are exchanged for those associated with a number of fields [8]. One benefit of this transformation is that the field theory lends itself well to various approximation schemes; most importantly, a mean-field approximation that produces the well-known equations of polymer selfconsistent field theory (SCFT). Physically, this approximation makes the assumption that there is a single "mean-field" configuration (a saddle point for the complex plane) that dominates the functional integrals comprising the partition function of the field theory - effectively ignoring fluctuations about the saddle point configuration. This mean-field SCFT approach can be justified by a formal steepest descent procedure for concentrated polymer fluids at high molecular weight and represents a significant simplification over the full theory. While still challenging numerically, the SCFT field equations have been used successfully to study a plethora of systems, such as block and graft copolymers, concentrated polymer solutions, thin polymer films, and

*This work was supported by NSF IGERT Grant DGE02-21715, NSF DMR-CMMT Grant 0603710, and Donors of the ACS administered Petroleum Research Fund (EML and GHF), NSF DMS Grant 0609996 (GOM and HDC), and NSF DMS Grant 0505738 (CJGC).

${ }^{\dagger}$ Department of Chemical Engineering, University of California, Santa Barbara, CA 93106-5080 (emlennon@engr.ucsb.edu).

${ }^{\ddagger}$ Department of Mathematics, University of California, Santa Barbara, CA 93106-5080 (gmohler@math.ucsb.edu).

${ }^{\S}$ Department of Mathematics, University of California, Santa Barbara, CA 93106-5080 (hdc@math.ucsb.edu).

IDepartment of Mathematics, University of California, Santa Barbara, CA 93106-5080 (cgarcia@math.ucsb.edu).

" Departments of Chemical Engineering \& Materials and Materials Research Laboratory, University of California, Santa Barbara, CA 93106-5080 (ghf@mrl.ucsb.edu). 
polymer blends and alloys [19, 21, 20, 28].

The natural limitations of SCFT are evident for a variety of systems. For example in the case of melts, the mean-field approximation breaks down for block copolymers near their order-disorder transition. In these cases, field fluctuations about the relevant saddle points cannot be neglected as they make important contributions to thermodynamic properties and self-assembly behavior. For example, in symmetric diblock copolymer melts, the fluctuations not only suppress the order-disorder temperature, but actually change the nature of the transition from continuous (second order) to discontinuous (first order) [10].

The limitations of SCFT are even more acute in dilute and semi-dilute polymer solutions, where strong excluded volume correlations (manifested by short-ranged field fluctuations) lead to significant departures from the predictions of mean-field theory for both structural (e.g. scattering functions) and thermodynamic (e.g. osmotic pressure) properties [5]. For this broad and technologically important class of polymeric liquids a quantitative treatment necessitates a return to the full field theory model and an alternative numerical approach.

Numerical investigations of the full field theory require an efficient method for importance sampling of the phase space spanned by the field variables. That is, the methods of simulating these systems must be designed to sample the areas in phase space which contribute the most to the properties of the system, rather than uniformly investigate every point in phase space. In particle-based models of classical equilibrium statistical mechanics, the Hamiltonian of the system is purely real, so such sampling can be effectively done with standard stochastic techniques such as Monte Carlo or real Langevin simulations. The corresponding polymer field theories, however, are characterized by complex Hamiltonians for which purely real sampling techniques are ill-suited. In particular, rapid phase oscillations associated with these methods lead to extreme difficulties when simulating large systems (the so-called "sign problem") [6, 8]. One method that has been shown to successfully bypass the sign problem for systems with a complex Hamiltonian is the complex Langevin $(C L)$ technique $[13,25]$. The method involves extending the field variables to the complex plane and writing an asymmetric stochastic Langevin dynamics for the real and imaginary components of the field. For select classes of complex Hamiltonians it can be proven that if the CL stochastic process converges to a steady state, then field configurations obtained by integrating the complex Langevin equations under stationary conditions can be used to importance sample the complex distribution of the full theory $[14,17]$. While no rigorous proof exists that the CL process has a stationary statistical distribution, this requirement can be tested numerically during the course of a simulation.

The CL approach was first applied to a field theory model of polymeric fluids by Ganesan and Fredrickson [12]. Beyond this initial study of a model for block copolymer melts, it was used to investigate models of semi-dilute polymer solutions [1] and ternary polymer alloys [7]. Despite these promising results, the CL equations are numerically challenging stochastic differential equations that combine stiffness with nonlocal and nonlinear force terms. Prior to this work, the numerical algorithms utilized for CL simulations have been explicit, low-order time integration schemes that suffer from poor stability and accuracy. This has limited the size and scope of systems for which field-theoretic polymer simulations can be applied and has been a major impediment to widespread adoption of the technique.

In this article, we develop semi-implicit strategies for integrating the complex 
Langevin equations that dramatically improve both stability and accuracy over loworder explicit schemes. We further generalize the CL equations to include colored noise and dissipation and exploit this generalization to affect Fourier acceleration. Beyond the immediate application of our improved algorithms to a broad class of polymer field theory models, we expect that our methods can be extended to CL simulations in very different physical contexts including correlated electron physics, time-dependent quantum chemistry, quantum chromodynamics, and lattice gauge theory.

The outline of the article is as follows: in Section 2 we describe our model system of a diblock copolymer melt and present the complex Langevin equations. We outline the numerical methods used to solve the stochastic CL differential equations obtained by spectral collocation with a plane wave basis and periodic boundary conditions in Section 3. In Section 4 we compare the performance of various algorithms outlined in the previous section. We conclude in Section 5 with a summary of our results and an outlook for the application of the complex Langevin simulations to study broader classes of polymer field theory models.

2. Diblock Copolymer Model. For demonstration purposes, we focus on implementing field-theoretic simulations of an incompressible model of a symmetric diblock copolymer melt. In 1980, Leibler developed a mean-field theory to study this system and described a second order phase transition between an ordered lamellar phase and a disordered phase [18]. Fredrickson and Helfand later showed that composition fluctuations induce a first order character to this order-disorder phase transition [10]. More recently, their findings were reproduced numerically using field-theoretic simulations in two dimensions [12]; three-dimensional simulations have not been computationally tractable hitherto. In this paper, we adopt the block copolymer model and notations introduced in a recent review article and book on the subject $[8,9]$.

We consider a canonical ensemble consisting of an incompressible melt of $n \mathrm{AB}$ diblock copolymers contained within a volume $V$. Each polymer has an A-block with a volume fraction $f$ of $A$-type statistical segments, or monomers. All numerical simulations were conducted for the special case of symmetric diblocks, for which $f=1 / 2$. The length $b$ and volume $v$ of each $\mathrm{A}$ and $\mathrm{B}$ segment is assumed to be the same, and the interactions between chemically dissimilar segments (relative to interactions among like segments) is characterized by a Flory parameter, $\chi$. A standard coarsegrained description of each copolymer is the continuous Gaussian chain model, which treats the polymer as a flexible thread of length $N$, where $N$ is its index of polymerization. A natural length scale of the system is the (unperturbed) radius-of-gyration of the polymer, $R_{g 0}=\left(N b^{2} / 6\right)^{1 / 2}$, which is a physical observable.

For the above model, a particle-to-field transformation [9] leads to a statistical field theory for the canonical partition function $Z$. This object can be expressed as a functional integral over two fluctuating chemical potential fields

$$
Z=\int \mathcal{D} W_{+} \int \mathcal{D} W_{-} \exp \left(-H\left[W_{ \pm}\right]\right)
$$

in which $W_{+}(\mathbf{r})$ and $W_{-}(\mathbf{r})$ are potential fields conjugate, respectively, to total segment density and to the difference between the densities of A and B segments. The Hamiltonian for the model system is given by

$$
H\left[W_{ \pm}\right]=C \int d \mathbf{r}\left[-i W_{+}+\frac{W_{-}^{2}}{\chi N}\right]-C V \ln Q\left[W_{ \pm}\right],
$$

where the common prefactor $C$ is a dimensionless chain concentration defined by $C=$ $n\left(R_{g 0}\right)^{3} / V$, and $Q\left[W_{ \pm}\right]$is the partition function (path integral) of a single polymer 
chain subject to the $W_{ \pm}$potential fields. We note that all lengths (or volumes) in the above expression have been made dimensionless by scaling with appropriate factors of $R_{g 0}$. The single-chain partition function can be evaluated according to

$$
Q\left[W_{ \pm}\right]=(1 / V) \int d \mathbf{r} q\left(\mathbf{r}, 1 ;\left[W_{ \pm}\right]\right)
$$

with the chain propagator, $q\left(\mathbf{r}, s ;\left[W_{ \pm}\right]\right)$, satisfying a Feynman-Kac formula [23]

$$
\frac{\partial}{\partial s} q(\mathbf{r}, s)=\nabla^{2} q(\mathbf{r}, s)-\psi(\mathbf{r}, s) q(\mathbf{r}, s), \quad q(\mathbf{r}, 0)=1 .
$$

In this modified diffusion equation the contour distance $s \in[0,1]$ along the chain is scaled by $N$, and the complex potential field $\psi(\mathbf{r}, s)$ is

$$
\psi(\mathbf{r}, s) \equiv \begin{cases}i W_{+}(\mathbf{r})-W_{-}(\mathbf{r}), & 0<s<f \\ i W_{+}(\mathbf{r})+W_{-}(\mathbf{r}), & f<s<1\end{cases}
$$

2.1. Mean-field Approximation. In the mean-field or self-consistent field (SCFT) approximation, one makes the assumption that there is a single set of $W_{ \pm}$"saddle point" field configurations that dominates the functional integral. It can be shown that in this mean-field approximation the Hamiltonian is proportional to the free energy of the system and thus must be a real number. As such, the $W_{+}$field will be purely imaginary whereas the $W_{-}$field will be real. Saddle-point solutions to this set of equations can then be found where the Hamiltonian is maximized with respect to the (real) $i W_{+}$field and minimized with respect to the $W_{-}$field. In general, such field theory models can possess multiple saddle points; the present diblock copolymer model has a homogeneous saddle point corresponding to the disordered phase, and inhomogeneous saddle points corresponding to ordered phases of different symmetry (lamellar, hexagonally-packed cylinders, body-centered cubic spheres, etc) [8]. For the symmetric case of $f=1 / 2$, only the disordered and lamellar saddle points are relevant to the phase behavior (i.e. they have the lowest values of free energy or $H$ ).

The relevant functional derivatives for locating saddle points are given as

$$
\begin{aligned}
& \frac{\delta H\left(W_{ \pm}\right)}{\delta W_{+}(\mathbf{r})}=i C\left[\phi_{A}(\mathbf{r})+\phi_{B}(\mathbf{r})-1\right] \\
& \frac{\delta H\left(W_{ \pm}\right)}{\delta W_{-}(\mathbf{r})}=C\left[-\phi_{A}(\mathbf{r})+\phi_{B}(\mathbf{r})+\frac{2}{\chi N} W_{-}(\mathbf{r})\right]
\end{aligned}
$$

where the dimensionless densities (volume fractions) of A and B type segments are

$$
\phi_{A}(\mathbf{r})=\frac{1}{Q} \int_{0}^{f} d s q(\mathbf{r}, s) q^{\dagger}(\mathbf{r}, 1-s)
$$

and

$$
\phi_{B}(\mathbf{r})=\frac{1}{Q} \int_{f}^{1} d s q(\mathbf{r}, s) q^{\dagger}(\mathbf{r}, 1-s)
$$

The backward propagator appearing in these expressions, $q^{\dagger}(\mathbf{r}, s)$, satisfies a second modified diffusion equation

$$
\frac{\partial}{\partial s} q^{\dagger}(\mathbf{r}, s)=\nabla^{2} q^{\dagger}(\mathbf{r}, s)-\psi^{\dagger}(\mathbf{r}, s) q^{\dagger}(\mathbf{r}, s), \quad q^{\dagger}(\mathbf{r}, 0)=1 .
$$


with

$$
\psi^{\dagger}(\mathbf{r}, s) \equiv \begin{cases}i W_{+}(\mathbf{r})+W_{-}(\mathbf{r}), & 0<s<1-f \\ i W_{+}(\mathbf{r})-W_{-}(\mathbf{r}), & 1-f<s<1 .\end{cases}
$$

The mean-field SCFT equations that determine the saddle point field configurations $W_{ \pm}^{*}$ correspond to

$$
\left.\frac{\delta H\left[W_{ \pm}\right]}{\delta W_{ \pm}(\mathbf{r})}\right|_{W_{ \pm}^{*}}=0
$$

Given that the parameter $C$ multiplies all terms in the Hamiltonian $H$, it is possible to show by a steepest descent analysis that the partition function is asymptotically dominated by the lowest energy saddle point configuration for $C \rightarrow \infty$. For threedimensional copolymer melts $C \sim N^{1 / 2}$, so it follows that the mean-field approximation (SCFT) is asymptotically valid for copolymers of very high molecular weight. Analysis of the SCFT equations for a symmetric diblock copolymer melt leads to the prediction of a second order phase transition from a disordered melt to a lamellar phase when $\chi N$ is increased above 10.495 [18].

2.2. Beyond mean-field: complex Langevin dynamics. Here we shall be interested not in mean-field solutions, but rather in full solutions of the statistical field theory that incorporate fluctuation effects. Our simulation tool will be the complex Langevin (CL) method [25], which is based on time integration of the following stochastic dynamics scheme for the coupled $W_{ \pm}$fields

$$
\frac{\partial W_{ \pm}(\mathbf{r}, t)}{\partial t}=-\lambda \frac{\delta H\left[W_{ \pm}\right]}{\delta W_{ \pm}(\mathbf{r}, t)}+\eta(\mathbf{r}, t)
$$

In these equations the variable $t$ is a fictitious time, rather than a physical one, and $\lambda>0$ is an arbitrary (real) relaxation parameter. It is further understood that both fields are extended throughout the complex plane so that the derivatives $\delta H / \delta W_{ \pm}$are taken as complex derivatives. The noise term $\eta(\mathbf{r}, t)$, in contrast, is purely real and is Gaussian and white in both space and time with the average properties

$$
\begin{aligned}
\langle\eta(\mathbf{r}, t)\rangle & =0, \\
\left\langle\eta(\mathbf{r}, t) \eta\left(\mathbf{r}^{\prime}, t^{\prime}\right)\right\rangle & =2 \lambda \delta\left(\mathbf{r}-\mathbf{r}^{\prime}\right) \delta\left(t-t^{\prime}\right) .
\end{aligned}
$$

The theoretical basis behind the complex Langevin method has been reviewed in $[8,13,25]$ and is beyond the scope of this paper. However, for a broad class of model Hamiltonians it can be shown that when these dynamics converge to a stationary distribution of states, averages computed with a Markov chain of states from CL trajectories under stationary conditions coincide with ensemble averages of the full field theory $[14,17]$.

More physically, we see that the CL equation (2.12) relaxes the fields in the direction of a complex force proportional to the derivative of the complex Hamiltonian. In the absence of the noise term $\eta$, these relaxational dynamics have stationary equilibrium points at saddle points of the model. With the noise term, the CL dynamics tends to drive the imaginary components of the fields towards a "constant phase" condition

$$
\Im\left(\frac{\delta H}{\delta W}\right)=\frac{\delta H_{I}}{\delta W_{R}} \approx 0,
$$


where subscripts $R$ and $I$ denote real and imaginary parts, respectively, of a complex function or field. The real part of equation (2.12), which contains the noise source, then serves to stochastically drive CL trajectories along this nearly constant phase path. The CL dynamics can thus be viewed as a type of adaptive stationary phase technique that can be applied to high dimensional problems and that requires no advance computation of saddle points or local analysis. In the present model, we note that as the parameter $C$ increases, the relative importance of the noise term is diminished so that the CL trajectories are restricted to narrow regions about one or more saddle points and the departure from mean-field behavior is small.

3. Numerical Methods. Simulating the complex Langevin equations in the framework of polymer field theory presents numerical hurdles in solving not only the stochastic differential equations, (2.12), but also the modified diffusion equations, (2.4) and (2.9). The coupled system requires accurate and stable methods that can address its inherent nonlinear and nonlocal nature. Thus the computational benefits gained by using a high order scheme for solving the complex Langevin equations may be limited without improved algorithms for solving the modified diffusion equations.

Previous work in field-theoretic simulations of polymers has focused primarily on numerical solutions in the mean-field (SCFT) approximation, i.e. on the problem of computing saddle points. Nevertheless, many of the advances made to algorithms in this limit can be modified for use beyond mean-field in the complex Langevin framework. For instance, the real and reciprocal space properties of the theory have been used to optimize relaxation schemes for the smooth fields characteristic of weakly segregated systems in the mean-field theory [20,2]. More recently, there has been a focus on simulating block copolymer systems in the strong segregation limit and with embedded nanoparticles in which the chemical potential fields are rapidly varying in space $[4,29]$. As the large gradients in these fields are similar to those created by the stochastic terms in the complex Langevin equations, we shall see that some of the recent developments in deterministic (mean-field) simulations can be extended to the stochastic non-mean-field case with good results. Despite the considerable advances in numerical methods for solving the SCFT equations, it is notable that prior to this work only the simplest explicit, first-order method (Euler-Maruyama) has been applied to solving the complex Langevin equations.

3.1. Diffusion Equations. An attractive method for solving the modified diffusion equations in cubic or parallelepiped domains subject to periodic boundary conditions is spectral collocation (pseudo-spectral) with a plane wave basis [15]. This method achieves spectral accuracy for smooth fields and allows for a diagonal representation of the linear operators appearing in the diffusion equations by treating the Laplacian in reciprocal space and the potential terms in real space. One popular approach in SCFT simulations is the operator splitting method of Rasmussen and Kalosakas and Tzeremes, Rasmussen, Lookman and Saxena [27, 30]. In this scheme, the formal solution to the diffusion equation is expanded to second order in contour step $\Delta s$ such that

$$
\begin{aligned}
q(\mathbf{r}, s+\Delta s) & =\exp \left[\Delta s\left(\nabla^{2}-\psi(\mathbf{r}, s)\right)\right] q(\mathbf{r}, s) \\
& \left.\left.=\exp \left[-\frac{\Delta s}{2} \psi(\mathbf{r}, s)\right)\right] \exp \left[\Delta s \nabla^{2}\right] \exp \left[-\frac{\Delta s}{2} \psi(\mathbf{r}, s)\right)\right] q(\mathbf{r}, s) \\
& +O\left(\Delta s^{3}\right)
\end{aligned}
$$


By using this second order accurate approach with fast Fourier transforms (FFTs) to convert from real to reciprocal space, each solution of the modified diffusion equation requires a computational effort that scales as $N_{s} N_{x} \log N_{x}$, where $N_{x}$ is the number of spatial collocation points and $N_{s}$ the number of contour steps. The solution of the diffusion equations indeed represents the most computationally demanding component of field-theoretic simulations.

In a recent paper, Cochran, García-Cervera and Fredrickson expanded the arsenal of pseudo-spectral methods by proposing an unconditionally stable BDF method to solve the modified diffusion equation for systems with sharp interfaces [4]:

$$
\begin{aligned}
& \frac{25}{12} q_{n+1}-4 q_{n}+3 q_{n-1}-\frac{4}{3} q_{n-2}+\frac{1}{4} q_{n-3}= \\
& \Delta s\left[\nabla^{2} q_{n+1}-\psi(\mathbf{r})\left(4 q_{n}-6 q_{n-1}+4 q_{n-2}-q_{n-3}\right)\right] .
\end{aligned}
$$

where the subscript denotes contour position, i.e. $q_{n}=q(\mathbf{r}, n \Delta s)$. To initialize this algorithm, the first three steps are taken using backward Euler stepping with Richardson extrapolation. The method is $4^{\text {th }}$ order accurate for smooth fields and damps high frequency modes making it ideal for stiff systems. Further, it is expected to exhibit $4^{\text {th }}$ order accuracy for any prescribed $W_{ \pm}$fields as long as $q$ is smooth in $s$.

Once the single chain propagator $q$ is determined for the given potential fields, the density operators, equations (2.7) and (2.8), are found locally using an appropriate scheme to integrate along $s$. We have focused on minimizing $N_{s}$ by using GaussLegendre quadrature, but other methods, such as the composite Simpson's method, are equally applicable.

3.2. Time Integration. The efficient evaluation of the complex Langevin equations (2.12) for each field requires accurate resolution of the time integration of the stochastic differential equation. Further, because this is a sampling method for an equilibrium statistical mechanics problem rather than a true dynamics trajectory, only a weakly convergent scheme is necessary [16]. Complicating matters is the fact that while the stochastic term is a purely additive Gaussian white noise, the force term is nonlinear. As such, previous complex Langevin simulations have been limited to a single step, explicit scheme. We show here that by using analytic features of the theory for weakly inhomogeneous fields, one can develop a class of methods that are more efficient, allow larger time steps, and are more accurate.

3.2.1. Euler-Maruyama (EM). A standard method for solving the complex Langevin equation is the stochastic analog to forward Euler time-stepping. As it requires only a single solution to the modified diffusion equation, the Euler-Maruyama (EM) algorithm is an attractive explicit scheme that is weakly convergent to order one [16]. For the present field-theoretic simulations, it takes the form

$$
W_{ \pm}^{\mathbf{r}, t+\Delta t}=W_{ \pm}^{\mathbf{r}, t}-\Delta t \lambda\left[\frac{\delta H\left[W_{ \pm}^{t}\right]}{\delta W_{ \pm}^{\mathbf{r}, t}}\right]+\eta^{\mathbf{r}, t}
$$

with

$$
\left\langle\eta^{\mathbf{r}, t}\right\rangle=0, \quad\left\langle\eta^{\mathbf{r}, t} \eta^{\mathbf{r}^{\prime}, t^{\prime}}\right\rangle=2 \lambda \frac{\Delta t}{\Delta V} \delta_{\mathbf{r}, \mathbf{r}^{\prime}} \delta_{t, t^{\prime}},
$$

and where $\Delta t$ is the time step and $\Delta V$ is the volume associated with a cell of the collocation grid. We note that the operations required in the update scheme of equation (3.3) are purely local in space (on the collocation grid); the nonlocality of the 
theory is manifest through the solutions of the diffusion equations that are required to evaluate the forces according to equations (2.6), (2.7), and (2.8). While it is simple to iterate through time and generate many configurational states with this algorithm, the method is ultimately unfeasible for large three-dimensional simulations as sampling uncorrelated field configurations is computationally demanding due to the small time step required for system stability. We note that the computational effort per time step of the Euler-Maruyama scheme is $O\left(N_{s} N_{x} \log N_{x}\right)$ since the solution of the diffusion equations and force evaluation dominate the calculation.

3.2.2. First Order Semi-Implicit (1S). An improvement to the explicit Euler updating used in mean-field calculations is a semi-implicit scheme introduced by Ceniceros and Fredrickson [2] which uses a first order expansion of the functional derivatives of the Hamiltonian in powers of the inhomogeneous parts of the fields. In the diblock melt, the terms of the expansion that have been shown to be stabilizing in the deterministic (SCFT) context are given as

$$
\begin{aligned}
{\left[\frac{\delta H}{\delta W_{+}}\right]_{l i n} } & =C\left(g_{A A}+2 g_{A B}+g_{B B}\right) * W_{+}, \\
{\left[\frac{\delta H}{\delta W_{-}}\right]_{l i n} } & =\frac{2 C}{\chi N} W_{-},
\end{aligned}
$$

where $f * h \equiv \int d \mathbf{r}^{\prime} f\left(\mathbf{r}-\mathbf{r}^{\prime}\right) h\left(\mathbf{r}^{\prime}\right)$ denotes a spatial convolution. The $g_{i j}$ are the Debye scattering functions [18] whose Fourier transforms are given by

$$
\begin{aligned}
& \hat{g}_{A A}(k)=\frac{2}{k^{4}}\left[f k^{2}+\exp \left(-k^{2} f\right)-1\right], \\
& \hat{g}_{A B}(k)=\frac{1}{k^{4}}\left[1-\exp \left(-k^{2} f\right)\right]\left[1-\exp \left(-k^{2}(1-f)\right)\right], \\
& \hat{g}_{B B}(k)=\frac{2}{k^{4}}\left[(1-f) k^{2}+\exp \left(-k^{2}(1-f)\right)-1\right] .
\end{aligned}
$$

A straightforward application of this force splitting approach to the complex Langevin update scheme is to treat the noise explicitly as in the Euler-Maruyama integration and the modified force term semi-implicitly. By adding the linearized force at the future time and subtracting the same term at the present time, the fields are updated according to

$$
\begin{aligned}
W_{ \pm}^{\mathbf{r}, t+\Delta t}=W_{ \pm}^{\mathbf{r}, t}-\Delta t \lambda\left[\frac{\delta H\left[W_{ \pm}^{t}\right]}{\delta W_{ \pm}^{\mathbf{r}, t}}\right. & +\left(\frac{\delta H\left[W_{ \pm}^{t+\Delta t}\right]}{\delta W_{ \pm}^{\mathbf{r}, t+\Delta t}}\right)_{l i n} \\
& \left.-\left(\frac{\delta H\left[W_{ \pm}^{t}\right]}{\delta W_{ \pm}^{\mathbf{r}, t}}\right)_{l i n}\right]+\eta^{\mathbf{r}, t}
\end{aligned}
$$

with the noise properties in (3.4). The extra computational cost of this modification over the Euler-Maruyama method is negligible, adding only a pair of Fourier transforms at every step.

While this first order semi-implicit (1S) method will be seen to offer increased stability over the Euler-Maruyama scheme in CL simulations, it is still only first order weakly. Moreover, because it relies on a weak inhomogeneity expansion to linearize the force the accuracy is expected to deteriorate as $C \rightarrow 0$. 
3.2.3. Second Order Splitting (2S). Once the force term is decomposed into its linear and nonlinear parts, the implementation of high order methods becomes more feasible. One such method that utilizes this form is the general second order splitting algorithm developed by Petersen and Öttinger $[24,26]$. This approach is analogous to the trapezoidal method used to solve deterministic differential equations. However, as only one term in the linearized force can be treated implicitly, the other must be estimated with an explicit method. In general, the Euler-Maruyama scheme is used as a first order approximation to the nonlinear terms at the future time. While the combination of this predicted value and the implicit linear term produces a second order weak approximation to the field profile, this method carries over the additional benefit of stability first seen in the semi-implicit method and derived from the nature of the added and subtracted linearized force term. For our model, this algorithm can be written

$$
\begin{aligned}
W_{ \pm}^{\tilde{t}}=W_{ \pm}^{t} & -\Delta t \lambda\left[\frac{\delta H}{\delta W_{ \pm}}\right]^{t}+\eta^{t} \\
W_{ \pm}^{t+\Delta t}=W_{ \pm}^{t} & -\frac{\Delta t \lambda}{2}\left[\left(\frac{\delta H}{\delta W_{ \pm}}\right)_{l i n}^{t+\Delta t}\right. \\
& \left.+\left(\frac{\delta H}{\delta W_{ \pm}}\right)^{\tilde{t}}-\left(\frac{\delta H}{\delta W_{ \pm}}\right)_{l i n}^{\tilde{t}}+\left(\frac{\delta H}{\delta W_{ \pm}}\right)^{t}\right]+\eta^{t}
\end{aligned}
$$

where the linearized terms are given by equation (3.5) and we have suppressed the dependence on the spatial grid point $\mathbf{r}$.

This method is second order in the weak sense, and it requires two solutions for each of the diffusion equations (2.4) and (2.9), one for the explicit estimation in the Euler step and a second to compute the force term $\left(\delta H / \delta W_{ \pm}\right)^{\tilde{t}}$ for the final step forward. This effectively doubles the computational effort per time step relative to the Euler-Maruyama and first order semi-implicit schemes. Further, because this second order splitting method $(2 \mathrm{~S})$ relies on the linear approximation of the force term, the stabilizing benefits may also decrease as $C$ becomes small. However, the $2 \mathrm{~S}$ method is expected to perform better than the $1 \mathrm{~S}$ method in small $\mathrm{C}$ simulations because it is fully second order weakly accurate.

A second higher order method that we considered was the stochastic semi-implicit backward differentiation formula (SSBDF) method recently developed by Ceniceros and Mohler [3]. The method is strongly second order for small noise amplitude, $\epsilon$, and it converges with strong order $O\left(\Delta t^{2}+\epsilon \Delta t+\epsilon^{2} \Delta t^{1 / 2}\right)$. Although it has shown promise in test simulations with large $C$, we are interested in methods that exhibit improvements over the entire range of $C$. As such, we will not discuss the method in this work.

3.2.4. Fourier Acceleration. Beyond the development of more robust time integration schemes, modifications of the complex Langevin equations to include spatially colored noise may result in algorithms better designed to tackle the multi-scale nature of polymer field theories. Fundamentally, the long time scales associated with long wavelength spatial modes hinder equilibrium sampling of the field theory by requiring prohibitively long simulations. At the other end of the spectrum, short wavelength spatial fluctuations tend to limit and control the stability of the simulations. 
Incorporating colored spatial noise into the complex Langevin equations consists of a change in the form of the relaxation constant $\lambda$ appearing both as a dissipative constant in front of the force term and as a constant prefactor in the variance of the noise. Specifically, we replace the relaxation constant $\lambda$ with a translationally invariant, real function $\lambda\left(\left|\mathbf{r}-\mathbf{r}^{\prime}\right|\right)$. This new function will appear as the kernel of a linear operator acting on the force in the generalized CL equation, and we demand that it be positive definite. The generalized complex Langevin equation can be written

$$
\frac{\partial W_{ \pm}(\mathbf{r}, t)}{\partial t}=-\int d \mathbf{r}^{\prime} \lambda\left(\left|\mathbf{r}-\mathbf{r}^{\prime}\right|\right) \frac{\delta H\left(W_{ \pm}\right)}{\delta W_{ \pm}\left(\mathbf{r}^{\prime}, t\right)}+\eta(\mathbf{r}, t),
$$

with the modified noise properties

$$
\begin{aligned}
\langle\eta(\mathbf{r}, t)\rangle & =0, \\
\left\langle\eta(\mathbf{r}, t) \eta\left(\mathbf{r}^{\prime}, t^{\prime}\right)\right\rangle & =2 \lambda\left(\left|\mathbf{r}-\mathbf{r}^{\prime}\right|\right) \delta\left(t-t^{\prime}\right) .
\end{aligned}
$$

It can be shown [8] that the above generalized CL equations with positive definite $\lambda(r)$ have the same characteristics as the original CL equations - namely that if the scheme converges to a steady state, it will properly importance sample the complex statistical weight of the full field theory.

Selection of the shape of $\lambda(r)$, or equivalently its spatial Fourier transform $\hat{\lambda}(k)$, is arbitrary subject to the requirement of positive definiteness, although it may be guided by both physical intuition and the behavior of the simulated system. In the original CL scheme, the function $\lambda(r)=\lambda \delta(\mathbf{r})$ is localized in space but is delocalized in reciprocal space, $\hat{\lambda}(k)=\lambda$, corresponding to white noise. We might imagine that by choosing a $\hat{\lambda}(k)$ that has a nonuniform distribution in reciprocal space (spatially colored noise), a more rapid exploration of phase space might be possible, leading to shorter equilibration times in CL simulations.

The stochastic differential equations for the generalized CL scheme can be solved with the same algorithms described previously for the original CL equations, the only change being that the convolution operator and noise terms in equation (3.9) are most efficiently evaluated in reciprocal space by taking an FFT.

4. Results. The performance of the newly developed algorithms was tested in a one-dimensional system with periodic boundary conditions in the $\mathrm{x}$-direction. This model is formulated in three dimensions, but only the $\mathrm{x}$-direction is represented numerically, so we assume uniformity in the y- and z- directions. All length scales are expressed in units of the unperturbed polymer radius of gyration, $R_{g 0}$, and the dimension of the system in the $\mathrm{y}$ - and z-directions are taken to be 1 . With these assumptions, $\Delta V=\Delta x$ and $C=n\left(R_{g 0}\right) / L$ where $\Delta x$ is the grid spacing and $L$ is the length of the system in the $\mathrm{x}$-direction.

The simulations were run on a Pentium $4,3.2 \mathrm{GHz}$ processor with $2 \mathrm{~GB}$ RAM under Linux. The primary code was written in C, and the standard FFTW packages were used for all Fourier transformations [11].

4.1. Diffusion Equation: Fixed Stochastic Field. Previous to this work, the algorithms presented for solving the diffusion equations for the chain propagators (i.e. equations (3.1) and (3.2)) have been tested only for smooth potential fields $W_{ \pm}$, such as are encountered in mean-field (SCFT) solutions of the field theory. In stochastic simulations of the full theory the fields become spatially rough, and it is important to understand the performance of the diffusion equation algorithms for 
(a)

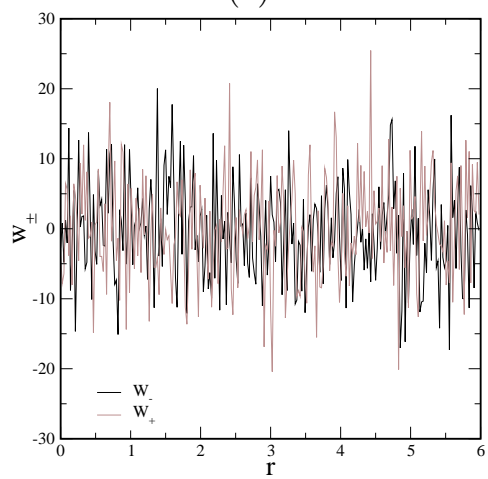

(b)

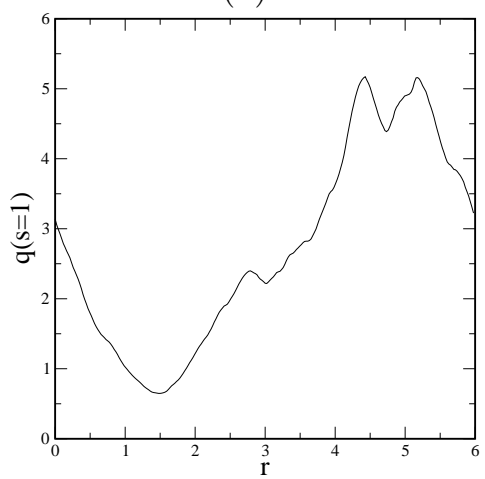

FIG. 4.1. (a) Random field configuration for $W_{ \pm}$fields and (b) the corresponding converged propagator profile for a field strength of $S=10$ and $\Delta s=10^{-4}$.

such stochastic fields, independent of the algorithms used for time stepping the CL equations. To this end, we have generated a single rough configuration of the $W_{ \pm}$ fields, and studied the convergence of the chain end propagator, $q\left(s=1, x ;\left[W_{ \pm}\right]\right)$, with respect to the number of contour steps $N_{s}$ at fixed spatial resolution $\Delta x$. We define an average contour step $\Delta s \equiv\left(N_{s}\right)^{-1}$, although in full CL simulations the local contour step $\delta s$ varies along the chain consistent with the Gauss-Legendre weights used in the quadrature for evaluating density operators. To mimic rough fields comparable to those seen in complex Langevin simulations, we set $W_{ \pm}(x)$ to $N(0, S)$, where $N(0, S)$ is a random number chosen (independently at each grid point $x$ ) from a Gaussian distribution centered at 0 and with a variance of $S$. The distribution of $N$ should have similar statistics to those of $\eta$, given in (3.4). In full CL simulations the noise strength has a variance $S=2 \Delta t /(C \Delta x)$, once the system has been discretized in space and time and we have made the convenient choice $\lambda=1 / C$. Typical values of $S$ range from $\ll 0.1$ to $>5$. In our diffusion equation tests, we chose an extreme value, $S=10$, which produces very rough field configurations for a system with $N_{x}=256$ and $L=6$. A sample set of $W_{ \pm}$field configurations and the resulting converged propagator profile $q\left(s=1, x ;\left[W_{ \pm}\right]\right.$) (using $\Delta s=10^{-4}$ ) are shown in Figure 4.1.

In Figure 4.2, we show the error obtained from the BDF and operator splitting methods as defined by $E_{q} \equiv \max _{i}\left|q\left(1, x_{i} ; \Delta s=10^{-4}\right)-q\left(1, x_{i} ; \Delta s\right)\right|$. The error of the BDF method is significantly smaller than that of the operator splitting method for the entire range of $\Delta s$ values considered. Further, each method is converging to roughly the expected order of 4 for $\mathrm{BDF}$ and 2 for operator splitting. When taking the function $q$ to be exact at $\Delta s=10^{-4}$, the operator splitting method requires more than 1000 contour steps to achieve 99\% accuracy. The BDF method achieves the same accuracy with an order of magnitude fewer contour steps, $N_{s} \approx 100$. Table 4.1 shows the order of each method for larger contour step sizes as calculated by the rate of convergence of the chain end propagator error as well as the CPU time required per solution of the diffusion equation using $N_{x}=256$ spatial points and $L=6$. While the BDF method is up to $70 \%$ slower than the operator splitting method, it has already reached its asymptotic order of 4 by $N_{s}=200$, whereas the operator splitting method has not entered its asymptotic order regime at the same contour resolution. 


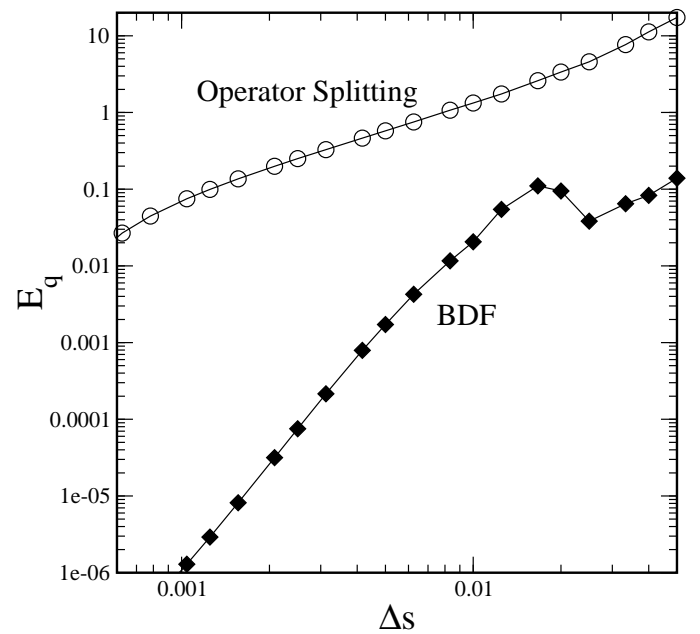

FIG. 4.2. Maximum error of the chain end propagator $q(1, x)$ as a function of $\Delta s$ for the BDF (3.2) and operator splitting (3.1) methods at a fixed $\Delta x=0.023$.

TABLE 4.1

Performance of BDF and operator splitting pseudo-spectral methods for low resolution propagator simulations.

Operator Splitting

$\mathrm{BDF}$

\begin{tabular}{|c||c|c||c|c|}
\hline$N_{s}$ & CPU time(sec) & Order & CPU time(sec) & Order \\
\hline 50 & 0.0289 & 1.44 & 0.0486 & 1.97 \\
100 & 0.0584 & 1.21 & 0.0881 & 3.56 \\
200 & 0.1185 & 1.11 & 0.1645 & 4.00 \\
\hline
\end{tabular}

4.2. Diffusion Equation: Full CL Simulations. Having determined the convergence rate of the chain propagator for a single realization of the potential fields, we now turn to study the effect of the chain resolution $\Delta s$ on the accuracy of averages computed from simulated trajectories of the CL equations. This is essentially a test of the diffusion equation algorithms. The only other place the contour resolution plays a role is in the quadrature schemes used to compute the density operators, but these schemes are higher order than the diffusion equation algorithms. In full CL simulations, the convergence of the chain propagator $q$ is not measured directly, but rather the expectation value of an observable, such as the Hamiltonian, is of interest. The value of $H$ is an especially important quantity in mean-field calculations (with smooth fields) as it corresponds to the free energy of the system. Beyond the meanfield approximation, the average Hamiltonian computed in a CL simulation can be coupled with a thermodynamic integration scheme to determine the free energy of a fluctuating system.

To investigate contour discretization effects in the full CL context, we ran a series of simulations to determine the contour resolution $\Delta s$ necessary to achieve a desired accuracy in the average of the real part of the Hamiltonian, $H_{R}=\Re(H)$, over the course of a CL trajectory. The average can be defined by either the ensemble average or the time average. To compute ensemble averages, a random initial condition for the $W_{ \pm}$fields is satisfied, and the system integrated forward to a set time, $t_{f}$, using CL dynamics. The process is repeated for multiple white noise seeds while applying 
the same initial conditions for the fields, and the convergence of the method with respect to $\Delta t$ is examined by constructing the ensemble average of $H\left(t=t_{f}\right)$ for each $\Delta t$. This ensemble average is defined

$$
\bar{H}_{R}=\frac{1}{N_{j}} \sum_{i=1}^{N j} H_{R}^{i}\left(t_{f}\right),
$$

where $N_{j}$ is the number of simulation runs (random seeds), and $H_{R}^{i}\left(t_{f}\right)$ is the real part of the Hamiltonian of simulation $i$ at time $t_{f}$.

Time averages are determined by running a single simulation and averaging the value of $H(t)$ under stationary conditions over a suitably broad time window. The average is defined as

$$
\left\langle H_{R}\right\rangle=\frac{1}{N_{p}} \sum_{n=1}^{N_{p}} H_{R}(n \Delta t),
$$

where $N_{p}$ is the number of time steps taken along the CL trajectory after the system has equilibrated and $N_{p} \Delta t$ is the width of the time window.

For the purpose of these tests, we used the first order semi-implicit time integration scheme with timestep $\Delta t=0.05$. The trajectories consisted of 40,000 iterations for each $\Delta s$, and we chose parameters corresponding to a strongly fluctuating, disordered copolymer melt: $C=100, \chi N=10$, and $L=4$. Here we focus on the relative performance of the two diffusion equation algorithms using a single CL time integration scheme. In a subsequent section we will discuss the relative performance of the three stochastic integration methods.

At a fixed spatial resolution $\Delta x$, we have found that the expectation value of the real part of $H,\left\langle H_{R}\right\rangle$, converges to a finite value for $\Delta s \rightarrow 0$. In Figure 4.3, we show the contour resolution $\Delta s$ required to achieve $0.1 \%$ and $0.5 \%$ error in $\left\langle H_{R}\right\rangle$ using the BDF diffusion equation algorithm as a function of the spatial resolution $\Delta x$. For high spatial resolution simulations, the necessary contour resolution for the BDF method scales (remarkably!) as $\Delta s \sim \sqrt{\Delta x}$, whereas for lower spatial resolution the relationship is linear. Interestingly, the operator splitting scheme does not perform so well in this "acid test" of diffusion equation algorithms. The operator splitting method requires a contour resolution that scales as $\Delta s \sim(\Delta x)^{\mu}$ with the exponent $\mu$ between 1 and 2 over the same range of $\Delta x$.

The implications of these results for the computational requirements of CL simulations are significant. A diblock melt at moderate interaction strength, e.g. $\chi N<20$, is generally resolved to $\Delta x=0.2$ or smaller. At this spatial resolution, the number of contour steps $N_{s}$ needed for accurate evaluation of the Hamiltonian using the BDF method scales linearly with the number of spatial collocation points $N_{x}$. On the other hand, the operator splitting method generally requires $N_{s} \sim N_{x}^{2}$. Thus by simply changing the algorithm for solving the modified diffusion equations to a method that is only modestly more expensive per contour step (BDF scheme), one can dramatically decrease the number of contour steps required by a large factor of order $N_{x}$.

4.3. Stochastic Integration: Ensemble Averages. Using a segregation strength and $\mathrm{C}$ value that corresponds to a strongly fluctuating but disordered copolymer melt, $\chi N=10$ and $C=100$, we have numerically explored the weak convergence characteristics of the three stochastic integration methods described. Specifically, we first 


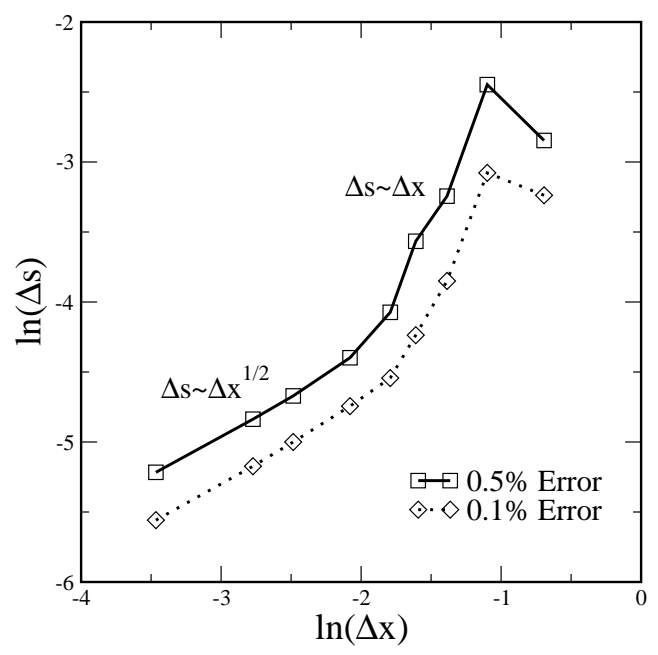

FIG. 4.3. Contour resolution requirements for the error in the Hamiltonian to be less than $0.5 \%$ (solid line) and $0.1 \%$ (dotted line) as a function of $\Delta x$ for a symmetric diblock with $C=100$ and $\chi N=10$ using the BDF scheme.

consider the weak convergence of the average Hamiltonian by looking at ensemble averages, as defined in (4.1). The accuracy of the method is then determined as a function of $\Delta t$ by comparing the value of the ensemble average computed for a given step size, $\bar{H}_{R}\left(\Delta t ; t_{f}\right)$, to an accurate reference value defined as $\bar{H}_{R}\left(\Delta t=0.5 ; t_{f}\right)$ calculated with the $2 \mathrm{~S}$ method.

In applying these stochastic integration schemes to polymer field theoretic simulations, we have found that as the fictitious time, $t$, increases the number of exploding trajectories increases. Despite these unbounded trajectories, approximations to the physical averages can still be made by rejecting those paths which are unbounded [22].

Table 4.2 lists the ensemble average $\bar{H}_{R}(t=80)$ for 3 different values of $\Delta t$ using each algorithm. The system parameters are $\chi N=10, C=100, L=8$, $N_{j} \Delta t=20,000, N_{x}=128$, and $\Delta s=(\Delta x)^{2}$. The trends show that the $2 \mathrm{~S}$ method is accurate up to the point when the number of exploding trajectories becomes non-zero. The 1S algorithm, on the other hand, converges with roughly order 1 and is has no exploding trajectories at any step size, but it is accurate for only very small values of $\Delta t$. As expected, the EM method is not accurate for modest values of $\Delta t$.

These trends can be further understood by the realization that the short simulation paths employed allow for oscillatory trajectories to be included in the ensemble averages. This effectively increases the error in the average Hamiltonian for any given set of simulation parameters. For example, the error of the EM method for $\Delta t=20.0$ is $\sim O\left(10^{2}\right)$, though $99 \%$ of trajectories are bounded. As $t_{f}=80$, however, this implies that the system is updated only 4 times. Hence the trajectory may oscillate without exploding.

4.4. Stochastic Integration: Time Averages. Calculating the time average of a given system is generally preferred to taking the ensemble average, as the latter requires the system be re-initialized $N_{j}$ times. As such, we focus now on the performance of the stochastic integration algorithms with respect to time averaging, as defined in (4.2). 
TABLE 4.2

Ensemble averages $\bar{H}_{R}(t=80)$ of the real part of the Hamiltonian for the Euler-Maruyama (EM), first order semi-implicit splitting (1S) and the second order semi-implicit splitting (2S) methods for multiple step sizes. The \% Bound column indicates the percentage of the $N_{j}=20,000$ simulations that did not explode for the step size. An accurate reference value, taken with the $2 S$ method at $\Delta t=0.5$, is $\bar{H}_{R}(t=80)=0.1195$.

\begin{tabular}{|c||c|c||c|c||c|c|}
\hline \multicolumn{1}{|c}{ EM } & \multicolumn{2}{c|}{$1 \mathrm{~S}$} \\
\hline 2.0 & $H_{R}$ & $\%$ Bound & $H_{R}$ & $\%$ Bound & $H_{R}$ & $\%$ Bound \\
\hline 8.0 & -65.39 & $100 \%$ & 0.103 & $100 \%$ & 0.119 & $100 \%$ \\
20.0 & 27.52 & $93 \%$ & 0.075 & $100 \%$ & -17.14 & $94 \%$ \\
\hline
\end{tabular}

(a)

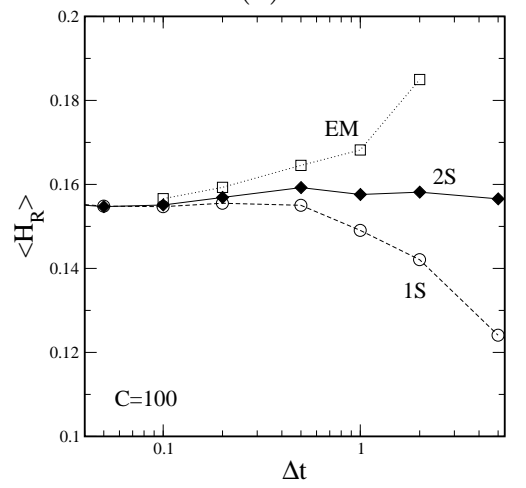

(b)

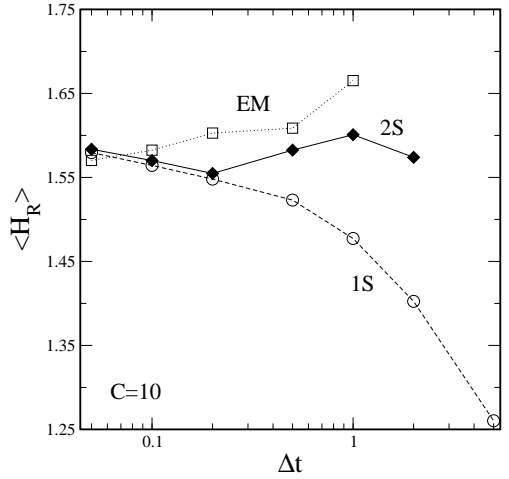

FIG. 4.4. Convergence of the real part of the Hamiltonian as $\Delta t$ decreases for the EulerMaruyama (EM), first order semi-implicit splitting (1S) and second order (semi-implicit) splitting (2S) methods for (a) $C=100$ and (b) $C=10$. The number of steps taken for each $\Delta t$ is adjusted such that the sampling error is smaller than the time integration for all points. The data from the $E M$ and $2 S$ schemes are limited at large $\Delta t$ by stability.

In Figure 4.4, we show the time average of the real part of the Hamiltonian, $\left\langle H_{R}\right\rangle$, as a function of the stochastic integration method and the time step size for our model system at $\chi N=10, L=8, N_{p} \Delta t=20,000, N_{x}=128$, and $\Delta s=(\Delta x)^{2}$ for both $C=100$ (moderate fluctuations) and $C=10$ (strong fluctuations). Of the newly developed algorithms, the first order semi-implicit method (1S) shows fewer exploding trajectories independent of $C$. However, as the method is still first order, the error becomes significant for time steps $\Delta t$ larger than $\sim 0.1$. On the other hand, the accuracy in determining $\left\langle H_{R}\right\rangle$ is remarkably independent of the step size for the second order splitting scheme $(2 \mathrm{~S})$, but this scheme is somewhat limited in stability when compared to the first order semi-implicit method.

Integration using the Euler-Maruyama (EM) method appears to be as accurate as scheme $1 \mathrm{~S}$ when looking only at $\left\langle H_{R}\right\rangle$, but it has poor overall stability. The deviation of the mean of the imaginary part of the Hamiltonian, $H_{I}$, is particularly useful to detect this instability. As $H$ is a physical quantity, its expectation value must be purely real. However, CL sampling allows for a complex-valued Hamiltonian for any given realization of the fields. In Figure 4.5 we show the average of $H_{I}$ for the same simulations shown in Figure 4.4. For time steps satisfying $\Delta t>0.1$, the EulerMaruyama method leads to an average imaginary part of the Hamiltonian, $\left\langle H_{I}\right\rangle$, that is $O(1)$ (and growing with length of the trajectory), whereas the other methods show 
(a)

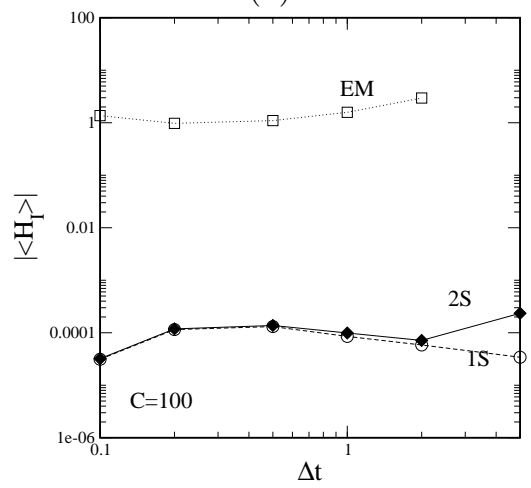

(b)

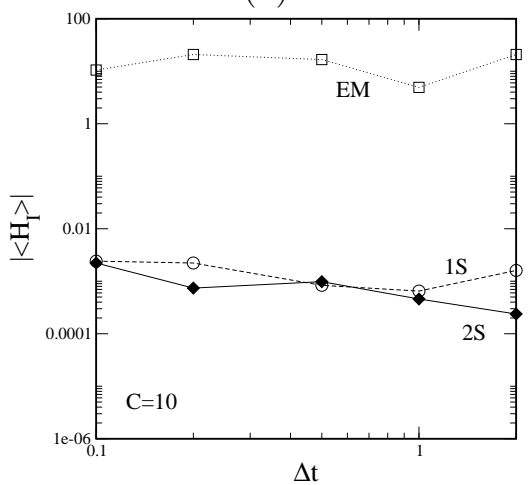

FIG. 4.5. The magnitude of the imaginary part of the Hamiltonian as a function of $\Delta t$ for each of the three stochastic integration methods for (a) $C=100$ and (b) $C=10$. The values have been averaged over the first $N_{p}$ steps where $N_{p} \Delta t=20,000$.

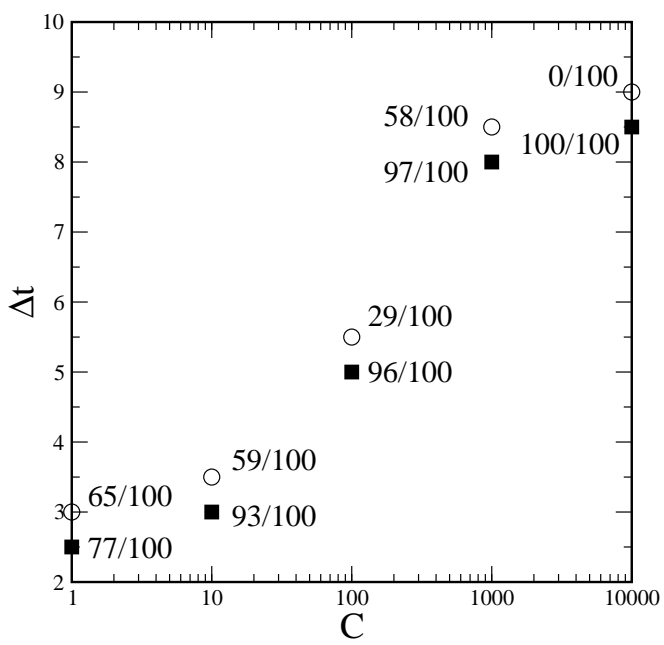

FIG. 4.6. Effect of $C$ on the stability of large time steps for the second order method 2S. Shown are the total number of bounded simulations per 100 runs. The squares (filled) are at the largest possible step size in which $<25 \%$ of the trajectories explode, and the circles (unfilled) are located at slightly larger step sizes, where $>25 \%$ of the trajectories explode.

the expected small values $\left\langle 10^{-4}\right.$ of $\left\langle H_{I}\right\rangle$ for these step sizes in a simulation where $C=100$. Similarly, when $C=10$ the average of $H_{I}$ is $O(10)$ after only 20,000 fictitious time steps, $N_{p} \Delta t=20,000$. The corresponding values calculated using the $1 \mathrm{~S}$ and $2 \mathrm{~S}$ methods are again $O\left(10^{4}\right)$ times smaller.

The practical choice of time step for each scheme depends on the stability of the method and accuracy desired. As the accuracy in $\left\langle H_{R}\right\rangle$ of the second order method $(2 \mathrm{~S})$ was shown to be nearly independent of the step size throughout its region of stability, stability is the limiting factor. We can thus characterize the performance of the $2 \mathrm{~S}$ method by determining the effect of the strength of the noise on the stability of simulations. For this purpose we have conducted a series of 1000 time step simulations using the $2 \mathrm{~S}$ stochastic algorithm and $N_{x}=64, L=8, N_{s}=150$, and $\chi N=10$. The noise strength parameter $C$ is varied systematically, and for each value of $C$ we 
(a)

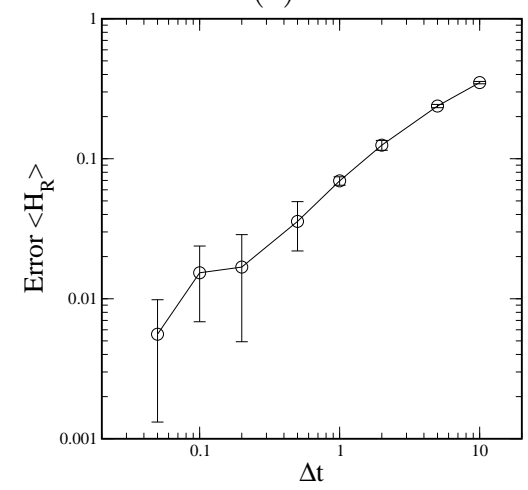

(b)

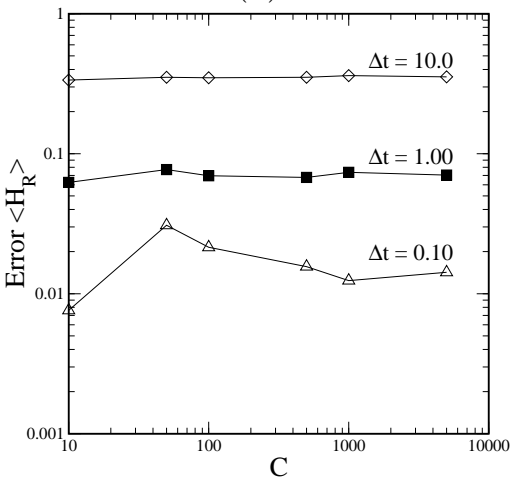

FIG. 4.7. Relative error in the real part of the Hamiltonian for algorithm $1 S$ (first order semiimplicit) as a function of (a) $\Delta t$ and (b) $C$. For the $\Delta t$ variations, each value is averaged over 9 different noise levels in the range $10 \leq C \leq 5000$, and the slope increases from $\sim 0.7$ (for large step sizes) to $\sim 0.8$ (for small step sizes) over the ranges of step sizes considered. The explicit dependence of the error as a function of $C$ is also shown for three selected step sizes.

determine the largest step size $\Delta t$ one can take while keeping the number of unstable exploding trajectories under 25\%. The results are shown in Figure 4.6. Each set of parameters was run 100 times, each from a different random number seed. For very low noise strength, $C>1000$, the stability limit plateaus around $\Delta t=8.5$, even as the noise level asymptotically approaches 0 . On the other hand, for highly fluctuating systems, $C<20$, the effect of $C$ on the stability is again small, generally requiring step sizes $\Delta t \lesssim 2$ for long simulations.

Unlike the second order method $2 \mathrm{~S}$, the first order semi-implicit method $1 \mathrm{~S}$ is extremely stable but of limited accuracy. Further, the relative (\%) error in the average real part of the Hamiltonian, $\left\langle H_{R}\right\rangle$, of any simulation using algorithm 1S appears to depend only on $\Delta t$ and not on $C$. This effect is captured explicitly in Figure 4.7 for a system with $N_{x}=64, L=8, N_{s}=150$, and $\chi N=10$. Each data point shown in the figure is averaged over 9 independent simulations with $C$ varying from 10 to 5000. The error is defined using the difference between $\left\langle H_{R}\right\rangle$ computed using scheme $1 \mathrm{~S}$ at the specified $\Delta t,\left\langle H_{R}\right\rangle_{1 S}$, and a reference accurate value of $\left\langle H_{R}\right\rangle$, $\left\langle H_{R}\right\rangle_{2 S}$, computed with scheme $2 \mathrm{~S}$ using $\Delta t=0.01$. The relative error, defined by $\left|\left\langle H_{R}\right\rangle_{1 S}-\left\langle H_{R}\right\rangle_{2 S}\right| /\left|\left\langle H_{R}\right\rangle_{2 S}\right|$, is then averaged over all $C$ values, and the results shown in Figure 4.7 (a). To understand the role of the noise strength parameter $C$ on the accuracy of simulations using scheme 1S, we show in Figure 4.7 (b) the relative error as a function of $C$ for three different $\Delta t$ values. Interestingly, the relative error for algorithm $1 \mathrm{~S}$ is approximately independent of the noise strength, and depends primarily on the size of the time step. The effect of $\Delta t$ on the error is consistent with a weakly first order method, as the slope in Figure 4.7 (b) is $\sim 0.75$ and increasing as $\Delta t$ decreases.

As each of the methods has been shown to converge to the same average value of $H_{R}$ for sufficiently small $\Delta t$, the best measure of the overall performance of each scheme is the CPU time required to reach a specified accuracy in $\left\langle H_{R}\right\rangle$. While the second order method $2 \mathrm{~S}$ is more accurate over a wide range of time steps, it requires twice the computational effort per step than the first order methods EM and 1S. Efficient simulations, however, must quickly sample uncorrelated points in phase space. 
TABle 4.3

Computational effort required to sample uncorrelated points using the first and second order methods $1 S$ and $2 S$ for $C=10$. The step size was chosen to be the largest possible while keeping the relative error below $2 \%$.

\begin{tabular}{|c|c|c|c|}
\hline Method & CPU time/step(sec) & $\Delta t_{\max }$ & CPU time(sec) \\
\hline $1 \mathrm{~S}$ & 0.032 & 0.1 & 32.16 \\
$2 \mathrm{~S}$ & 0.074 & 2.0 & 3.91 \\
\hline
\end{tabular}

The number of fictitious time steps between these points is the correlation time, $t_{C}$. This time is to a very good approximation independent of both the relaxation method employed in the simulation and the time step $\Delta t$. Moreover, $t_{C}$ plays a key role in determining the sampling error, which scales as $\sqrt{t_{C} / t}$, where $t=N_{p} \Delta t$ is the width of the sampling window used to compute time averages. Thus, under conditions where the algorithm $2 \mathrm{~S}$ allows for the use of a $\Delta t$ that is more than twice as large as the time step under scheme 1S for the same level of accuracy, we expect that the second order algorithm will outperform the first order scheme in reducing both CPU time and statistical sampling error.

To compare the relative performance of the $1 \mathrm{~S}$ and $2 \mathrm{~S}$ algorithms, we have determined the computation time required to reach a specific $t>t_{C}$ for a test system with $N_{x}=128, L=8, \Delta s=(\Delta x)^{2}, \chi N=10$, and $C=10$. In Table 4.3 we give the $\mathrm{CPU}$ time required to reach $t=100$ for the $1 \mathrm{~S}$ and $2 \mathrm{~S}$ methods while setting $\Delta t$ for each method to the largest value, $\Delta t_{\max }$, consistent with a relative error in $\left\langle H_{R}\right\rangle$ of less than $2 \%$ (c.f. Figure 4.4). While the second order method is more than twice as expensive as the first order semi-implicit method per step, the computation time required to obtain uncorrelated field configurations is reduced by an order of magnitude due to the significant difference in time step size.

4.5. Fourier Acceleration. As described previously, it is natural to expect that generalized CL schemes with colored spatial noise may be better suited than the conventional CL method to address the multi-scale features (in both space and time) of polymer field theory models. Defining a colored noise scheme requires specification of the function $\lambda(r)$, or correspondingly its spatial Fourier transform $\hat{\lambda}(k)$, which dictates the spatial pair correlation function of the noise. Optimal selection of this translationally invariant function is non-trivial, and various factors must be considered. For example, physically-inspired noise distributions, such as the noise spectrum associated with diffusive dynamics $\hat{\lambda}(k) \sim k^{2}$, drive the system most strongly at short scales/high wavenumbers. However, this same "conserved" dynamics implies that the relaxation of field variables is slowest at long length scales. The convergence of simulations is then delayed by the need to anneal the longest wavelength modes, particularly in ordered systems.

An appropriate choice of $\hat{\lambda}(k)$ will evidently be model dependent and possibly parameter dependent. For the present case of the symmetric diblock copolymer melt model, we exploit the fact that the equilibrium distribution of field fluctuations is strongly peaked about a spherical shell of non-zero wave vectors, $k_{m} \approx 3$, in reciprocal space [18]. As we shall see below, these modes are the slowest relaxing in the system, at least in the disordered melt phase. By choosing a colored noise spectrum that mimics the relaxation times of the fields in reciprocal space, an algorithm can be defined that relaxes all spatial Fourier modes at approximately the same rate. While we have no rigorous proof that such a noise spectrum is optimal, we provide numerical evidence 
(a)

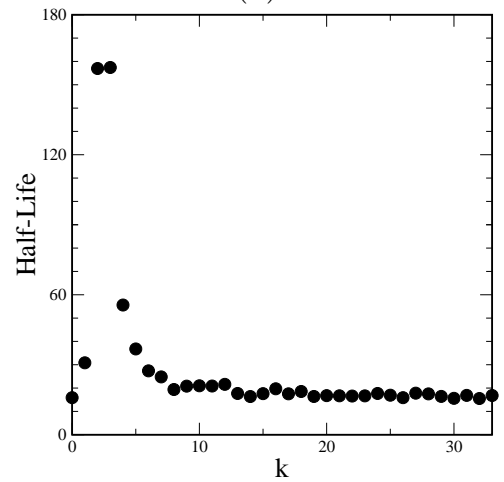

(b)

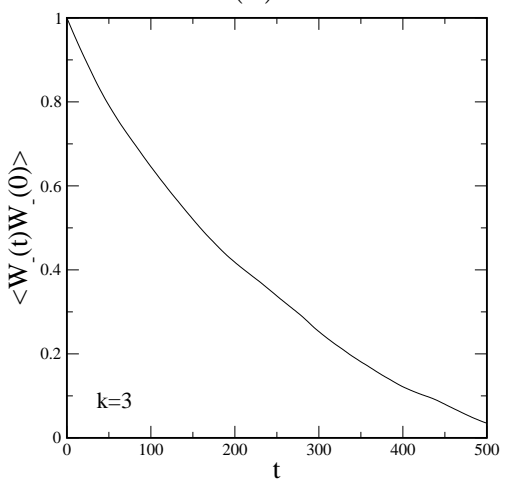

FIG. 4.8. The half-life (a) of the time correlation function of $\hat{W}_{-}$as a function of wave number. The time correlation function (b) for a single wave number, $k=3$.

below that it does appear to be an improvement over the standard white-noise based CL technique.

In Figure 4.8(a), we show the half-life of the relaxation for each Fourier mode of the exchange potential field $W_{-}$as defined by the time, $\tau$, that satisfies

$$
\frac{\left\langle\hat{W}_{-, R}(\mathbf{k}, \tau) \hat{W}_{-, R}(-\mathbf{k}, 0)\right\rangle}{\left\langle\hat{W}_{-, R}(\mathbf{k}, 0) \hat{W}_{-, R}(-\mathbf{k}, 0)\right\rangle}=0.5
$$

These results were obtained with a conventional white-noise CL scheme using algorithm $2 \mathrm{~S}$ and for parameters $\Delta t=0.1, N_{x}=64, L=8, N_{s}=150, \chi N=10$, and $C=100$. The second pressure-like field $W_{+}$fluctuates very quickly and, while generally responsible for inducing the main stiffness in the stochastic integration, has an insignificant correlation time. Thus, we focus our analysis on the fluctuation spectrum of the $W_{-}$field. While our test system is still within the disordered regime at $\chi N=10$, the length scale $\sim 2 \pi / k_{m}$, corresponding to the preferred domain size for the ordered phase, dominates the equilibrium fluctuation spectrum (equal time correlations) of the $W_{-}$field. Figure 4.8 (a) shows that the $W_{-}$Fourier modes with $k \equiv|\mathbf{k}| \approx k_{m}$ are also the slowest relaxing modes.

The observed $k$-dependence of the half life of $\hat{W}_{-}$modes closely mimics the $k$ dependence of the Debye function that enters the linearized force term for the $W_{-}$ field. While only the $\chi$ terms proved to be stabilizing in constructing the semi-implicit relaxation methods, see (3.5), the entire expansion of the force to first order in $W_{-}$is

$$
\frac{1}{C}\left[\frac{\delta H}{\delta W_{-}}\right]=\frac{2}{\chi N} W_{-}-\left(g_{A A}-2 g_{A B}+g_{B B}\right) * W_{-}+O\left(W_{+}\right)+O\left(W_{-}^{2}\right),
$$

where $g_{i j}$ are the Debye functions defined in Equation (3.6). Inspection of the function $\hat{g}_{T}(k) \equiv \hat{g}_{A A}(k)-2 \hat{g}_{A B}(k)+\hat{g}_{B B}(k)$ indicates that it is indeed strongly peaked near $k_{m} \approx 3$ and mirrors the $k$-dependence shown for the mode half-life in Figure 4.8(b). Because $\hat{\lambda}(k)$ enters the generalized CL equations both as a noise strength and mode relaxation rate, it would seem that the choice

$$
\hat{\lambda}(k)=\hat{g}_{T}(k),
$$




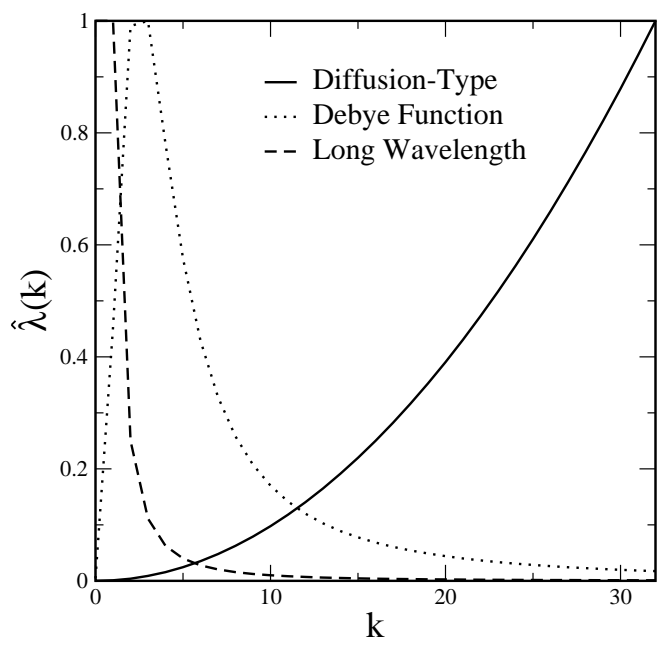

FIG. 4.9. Three different models for the colored spatial noise distribution $\hat{\lambda}(k)$ employed in generalized CL simulations of diblock copolymer melts. The distributions are non-negative and are normalized to unit maximum noise strength over the range of wave numbers used in the simulations.

is a rational way to approximately cancel the "critical slowing down" of the $k \approx k_{m}$ fluctuation modes observed in the white noise CL simulations.

Two other model colored noise distributions studied in this paper are simple diffusion-inspired noise and a long wavelength biased noise. The diffusion-type noise, given by $\hat{\lambda}(k)=k^{2}$, corresponds to the transform of the diffusive operator $-\nabla^{2}$ appearing in phase field models, such as the Cahn-Hilliard equations. Diffusive noise evidently relaxes high wave number fluctuations most rapidly, but also provides a strong source of roughness for the stochastic fields. While physically motivated, diffusive noise can limit the stability of stochastic integration schemes. The last colored noise model considered here is a long-wavelength biased noise that is the inverse of diffusion-type noise, $\hat{\lambda}(k)=k^{-2}$. Conceptually, the high wave number damping should improve stability, while the longest wavelength modes are relaxed most rapidly to (ideally) move the system quickly between metastable states. All three of these model noise spectra, shown in Figure 4.9, are scaled such that $\max _{k} \hat{\lambda}(k)=1$ for $k$ over the range of allowable wave numbers. We note that the $k=0$ uniform mode does not influence the thermodynamics of the model in the canonical ensemble, so fluctuations of this mode are explicitly suppressed.

Using purely white noise in the $W_{+}$field, and the three different types of colored noise in the $W_{-}$field, we studied the accuracy and relaxation times of $H_{R}$ for a system of $L=8, N_{x}=128$ and $N_{s}=256$ at $\chi N=10$ and $C=100$. The stochastic integration method employed was the first order scheme $1 \mathrm{~S}$ as it is the most stable algorithm. The effect of the colored noise spectrum on the accuracy of $\left\langle H_{R}\right\rangle$ is shown in Figure 4.10. The error bars are the deviation in the running average, each of which is taken over 5000 fictitious time steps, $N_{p} \Delta t=5000$. For all colored noise spectra and time step sizes $<10$ the accuracy is within $10 \%$ and is markedly better than the white noise model for $\Delta t>1$.

The decay time of the autocorrelation function of the real part of the Hamiltonian provides more insight into the mechanisms for this uniform improvement in accuracy. For each colored noise spectrum, the correlation time increases compared with that 


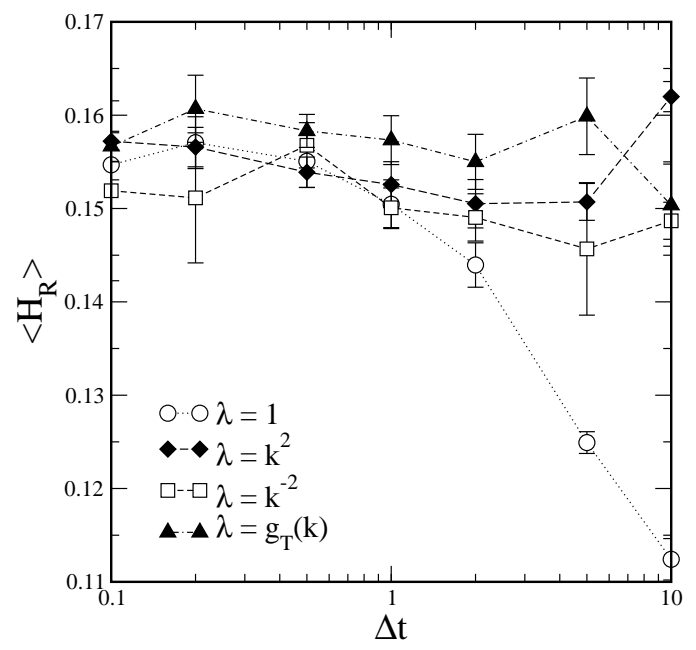

FIG. 4.10. The average value of the real part of the Hamiltonian as a function of the step size for the white noise $(\hat{\lambda}(k)=1)$ and three colored noise spectra considered for a system with $N_{x}=128, L=8, N_{s}=256, \chi N=10$ and $C=100$.

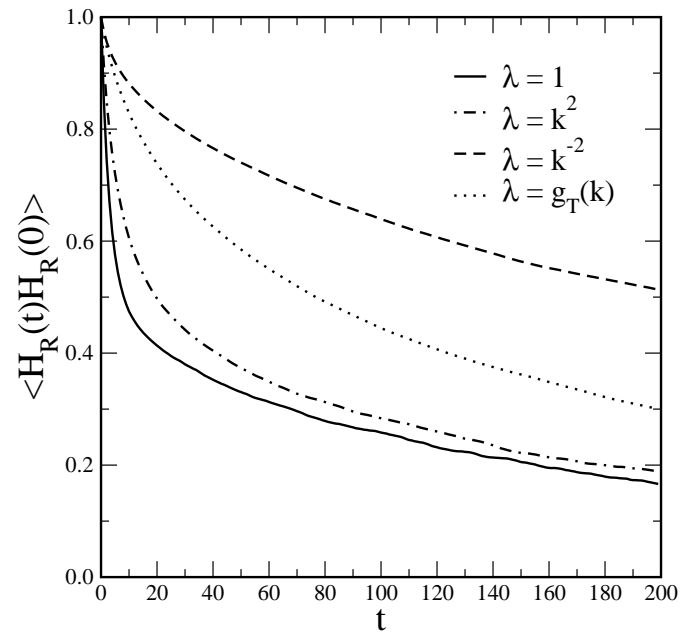

FIG. 4.11. The autocorrelation function of the real part of the Hamiltonian for the four noise spectra considered for a simulation with the parameters $N_{x}=128, L=8, \Delta t=1.0, N_{p}=2 \times$ $10^{4}, N_{s}=256, \chi N=10$ and $C=100$. The function has been normalized by the second moment of $H_{R}$.

for white noise as shown in Figure 4.11. For the Debye noise $\hat{g}_{T}(k)$, this increase is ten-fold. The diffusion and inverse diffusion noise spectra show increases of 2.5 and 15 times, respectively. This effect is presumed to come from scaling $\lambda$ to be 1 at its maximum, as the trends in correlation time mirror inversely the integrated areas of the noise spectra shown in Figure 4.9. At the same step size $\Delta t$, simulations with colored noise distributions $\hat{\lambda}(k)<1$ relax nearly all modes more slowly than the white noise model with $\hat{\lambda}(k)=1$.

The true test for the performance of a colored noise algorithm versus the white noise scheme is therefore to compare correlation times when each model is simulated 
TABLE 4.4

Computational requirements for each of the noise spectra considered using the $1 S$ scheme. The maximum time step, $\Delta t_{\max }$, is the largest step that can be taken while ensuring that $\left\langle H_{R}\right\rangle$ is within $5 \%$ of the accurate reference value computed with the $2 S$ scheme using $\Delta t=0.01$. The correlation time $\tau_{C}$ is computed as the half-life from the autocorrelation function of $H_{R}$.

\begin{tabular}{|c|c|c|c|c|}
\hline Noise Spectra & Function & $\tau_{C}$ & $\Delta t_{\max }$ & CPU time $/ \tau_{C}(\mathrm{sec})$ \\
\hline White & $\hat{\lambda}(k)=1$ & 9 & 1.0 & 0.289 \\
Debye & $\hat{\lambda}(k)=\hat{g}_{T}(k)$ & 77 & 20 & 0.123 \\
Diffusion & $\hat{\lambda}(k)=k^{2}$ & 20 & 10 & 0.064 \\
Inverse Diffusion & $\hat{\lambda}(k)=k^{-2}$ & 214 & 0.5 & 13.376 \\
\hline
\end{tabular}

at the maximum time step consistent with a prescribed accuracy. For example, the average Hamiltonian of the Debye noise model is still accurate to within $5 \%$ for step sizes $\Delta t>20$. While the correlation time of this model is a factor of ten larger than the white noise model, the twenty-fold gain in step size for the same accuracy over the white noise simulation means that Debye colored noise provides a two-fold improvement in terms of computational requirements. Table 4.4 summarizes this comparison in performance using the $1 \mathrm{~S}$ algorithm for all three colored noise models against the white noise model.

Interestingly, the diffusive model considerably outperforms the white noise model and the other two colored noise models. Both the Debye and diffusion models perform better than the conventional white noise CL scheme. While these results are promising, they should be considered as preliminary and should be extended to a wider variety of parameters and polymeric fluid models.

5. Conclusions. There is great interest in the development of numerical methods for simulating classical statistical field theory models of inhomogeneous polymeric fluids - so called "field-theoretic polymer simulations." These models are plagued by the sign problem associated with complex Hamiltonians, but the complex Langevin (CL) simulation technique has been shown to provide an effective numerical tool for circumventing this problem. Nonetheless, the stochastic CL equations present a formidable set of nonlinear, non-local field equations that have not been previously the subject of detailed study.

In the present paper, we have shown that analytic information about the linearized complex force that drives the dynamical evolution can be used to develop effective semi-implicit time integration schemes for the CL equations. These schemes have significantly better stability than the simplest explicit Euler-Maruyama method, and one of our algorithms, scheme 2S, adopted from a second order weak algorithm [24, 26], shows dramatically improved accuracy in application to a standard field theory model of block copolymer melts.

Another significant finding is that the use of higher-order schemes for resolving the chain propagator $q(\mathbf{r}, s)$ along the contour variable $s$, which is the most computationally demanding step of a field-theoretic polymer simulation, can significantly improve the performance of CL simulations. Specifically, we show that a fourth-order semi-implicit backwards differentiation scheme (BDF) can reduce the computational effort of a stochastic CL simulation by an order of magnitude when compared with standard second-order algorithms.

Finally, we have reported on a preliminary investigation of the use of a "general- 
ized" CL scheme that forces the CL equations with spatially colored noise, rather than the white noise conventionally applied in CL simulations. We find numerical evidence that the efficiency of CL simulations can indeed be improved by a proper selection of the colored noise spectrum. The strategy of attempting to select a noise spectrum $\hat{\lambda}(k)$ that makes the relaxation rate of field fluctuation modes with wave number $k$ more uniform seems to be effective at improving the performance of CL simulations. Nonetheless, we have no proof that this is an optimal strategy and much work remains to be done to establish the best algorithms and noise statistics for conducting CL simulations on a broad class of models.

By appropriately coupling the above methods, we have shown that field-theoretic simulations employing the stochastic complex Langevin method are a tractable solution to studying polymer physics on the mesoscale. We are optimistic that this work and further developments will allow the investigation of broad classes of important physical systems including block and graft copolymers, polymer alloys, polyelectrolytes, and liquid crystalline polymers.

Acknowledgments. The authors are grateful to E. Cochran and K. Katsov (Banc of America Securities, LLC) for many helpful discussions.

\section{REFERENCES}

[1] A. J. Alexander-Katz, A. G. Moreira, and G. H. Fredrickson, Field-theoretic simulations of confined polymer solutions, Journal of Chemical Physics, 118 (2003), pp. 9030-9036.

[2] H. D. Ceniceros And G. H. Fredrickson, Numerical solution of polymer self-consistent field theory, Multiscale Modeling and Simulation, 2 (2004), pp. 452-474.

[3] H. D. Ceniceros And G. O. Mohler, A Practical and Accurate Method for Stiff Stochastic Differential Equations with Small Noise, Multiscale Modeling and Simulation, 6 (2007), pp. $212-227$.

[4] E. W. Cochran, C. J. García-Cervera, and G. H. Fredrickson, Stability of the gyroid phase in diblock copolymers at strong segregation, Macromolecules, 39 (2006), pp. 24492451. E. W. Cochran, C. J. García-Cervera, and G. H. Fredrickson, Stability of the gyroid phase in diblock copolymers at strong segregation, Macromolecules, 39 (2006), p. 4264 .

[5] P. G. De Gennes, Scaling Concepts in Polymer Physics, Cornell University Press, 1979.

[6] H. De Raedt and A. LagendiJk, Monte Carlo Calculation of the Thermodynamic Properties of a Quantum Model: A One-Dimensional Fermion Lattice Model, Physical Review Letters, 46 (1981), pp. 77-80.

[7] D. Düchs, V. Ganesan, G. H. Fredrickson, and F. Schmid, Fluctuation effects in ternary $A B+A+B$ polymeric emulsions, Macromolecules, 36 (2003), pp. 9237-9248.

[8] G. H. Fredrickson, The Equilibrium Theory of Inhomogenous Polymers, Clarendon Press, 2006.

[9] G. H. Fredrickson, V. Ganesan, And F. Drolet, Field-Theoretic Computer Simulation Methods for Polymers and Complex Fluids, Macromolecules, 35 (2002), pp. 16-39.

[10] G. H. Fredrickson And E. Helfand, Fluctuation effects in the theory of microphase separation in block copolymers, Journal of Chemical Physics, 87 (1987), pp. 697-705.

[11] M. Frigo and S. G. Johnson, The Design and Implementation of FFTW3, Proceedings of the IEEE, 93 (2005), pp. 216-231.

[12] V. Ganesan and G. H. Fredrickson, Field-theoretic polymer simulations, Europhysics Letters, 55 (2001), pp. 814-820.

[13] J. R. KlaUder, A Langevin approach to fermion and quantum spin correlation functions, Journal of Physics A, 16 (1983), pp. L317-L319.

[14] H. Gausterer And S. Lee, The mechanism of complex Langevin simulations, Journal of Statistical Physics, 73 (1993), pp. 147-157.

[15] D. Gottlieb and S. A. Orszag, Numerical Analysis of Spectral Methods: Theory and Applications, Society for Industrial and Applied Mathematics (SIAM), 1977.

[16] P. E. Kloeden And E. Platen, Numerical Solution of Stochastic Differential Equations, Springer, 1991. 
[17] S. LEE, The convergence of complex Langevin simulations, Nuclear Physics B, 413 (1994), pp. 827-848.

[18] L. LEIBLER, Theory of microphase separation in block copolymers, Macromolecules, 13 (1980), pp. $1602-1617$.

[19] M. W. MATSEn, Stabilizing new morphologies by blending homopolymer with block copolymer, Physical Review Letters, 74 (1995), pp. 4225-4228.

[20] M. W. MatSen And M. Schick, Stable and unstable phases of a diblock copolymer melt, Physical Review Letters, 72 (1994), pp. 2660-2663.

[21] M. W. Matsen, The standard Gaussian model for block copolymer melts, Journal of Physics: Condensed Matter, 14 (2002), pp. R21-R47.

[22] G. N. Milstein and M. V. Tretyakov, Numerical Integration of Stochastic Differential Equations with Nonglobally Lipschitz Coefficients, SIAM Journal of Numerical Analysis, 43 (2005), pp. 1139-1154.

[23] B. K. ØKSENDAL, Stochastic Differential Equations: An Introduction with Applications, Springer, 2003.

[24] H. C. Öтtinger, Stochastic Processes in Polymeric Fluids, Springer-Verlag, 1996.

[25] G. PARISI, On complex probabilities, Physics Letters, 131B (1983), pp. 393-395.

[26] W. P. Petersen, A general implicit splitting for stabilizing numerical simulations of ito stochastic differential equations, Journal on Numerical Analysis, 35 (1998), pp. 1439-1451.

[27] K. Ø. RAsmussen AND G. Kalosakas, Improved numerical algorithm for exploring block copolymer mesophases, Journal of Polymer Science Part B, 40 (2002), pp. 1777-1783.

[28] F. Schmid, Self-consistent-field theories for complex fluids, Journal of Physics: Condensed Matter, 10 (1998), pp. 8105-8138.

[29] S. W. Sides, B. J. Kim, E. J. Kramer, and G. H. Fredrickson, Hybrid particle-field simulations of polymer nanocomposites, Physical Review Letters, 96 (2006), 250601.

[30] G. Tzeremes, K. Ø. Rasmussen, T. Lookman, and A. Saxena, Efficient computation of the structural phase behavior of block copolymers, Physical Review E, 65 (2002), 041806. 